\title{
Identification of cancer stem cells from hepatocellular carcinoma cell lines and their related microRNAs
}

\author{
YANGMEI XU, YUNQING XIE, XIANGRU WANG, XUEFANG CHEN, \\ QINGYIN LIU, MINGANG YING and QIUHONG ZHENG
}

\begin{abstract}
Fujian Provincial Key Laboratory of Tumor Biotherapy, Teaching Hospital of Fujian Medical University, Fujian Provincial Tumor Hospital, Fuzhou 350014, P.R. China
\end{abstract}

Received June 24, 2013; Accepted August 8, 2013

DOI: $10.3892 /$ or.2013.2703

\begin{abstract}
The aim of this study was to identify cancer stem cells (CSC) from three hepatocellular carcinoma (HCC) cell lines and to screen for specific microRNAs (miRNAs) regulating CSCs. Side population (SP) phenotype analysis was used. Four factors in the staining process, the incubation time, shaking interval, culture time and Hoechst 33342 concentration were explored, respectively, to define the SP subtype. CSC characteristics of SP cells were verified by sphere-forming assay and tumorigenic ability in NOD/ SCID mice. QPCR assay for 370 miRNAs was performed to identify the differential miRNA expression between SP and Non-SP (NSP) cells in the PLC/PRF/5 cell line. The selected miRNAs were tested again in SP and NSP cells from Huh-7 and Hep-3B cell lines by qPCR assay. All four factors influenced SP percentage, when the other three conditions were fixed, the optimal Hoechst 33342 concentrations determined were $11 \mu \mathrm{g} / \mathrm{ml}$ for PLC/PRF/5 cells, $4 \mu \mathrm{g} / \mathrm{ml}$ for Huh-7 and $5 \mu \mathrm{g} / \mathrm{ml}$ for Hep-3B cells. The resultant SP percentage was $0.73 \pm 0.12 \%, 0.49 \pm 0.04 \%$ and $0.63 \pm 0.08 \%$, respectively. The purity of sorted SP cells was $>85 \%$. Floating spheres were formed by SP cells from all three cell lines, while NSP cells did not form a single floating sphere. Mice injected with SP cells on the right side formed more tumor masses compared to their counterpart NSP at the same injection dosage; qPCR profiling identified 27 differentially expressed miRNAs in PLC/PRF/5 cells. Subsequent qPCR assay showed that miR-9* and miR-194 were also downregulated in SP cells from Huh-7 and Hep-3B. The present study identified CSCs via SP and sphere-forming assay from three liver cancer cell lines. Altogether, 27 CSC-specific miRNAs were determined
\end{abstract}

Correspondence to: Professor Qiuhong Zheng, Fujian Provincial Key Laboratory of Tumor Biotherapy, Teaching Hospital of Fujian Medical University, Fujian Provincial Tumor Hospital, Fuma Road 420, Fuzhou 350014, P.R. China

E-mail: zqh2858@foxmail.com

Key words: cancer stem cells, side population cells, hepatocellular carcinoma, microRNA, floating spheres in PLC/PRF/5; miR-9* and miR-194 were identified as the common CSC-specific miRNAs across the three HCC cell lines.

\section{Introduction}

Recent studies continue to support the CSC hypothesis in which CSC is claimed to be the root of carcinogenesis. The existence of liver CSC has also been validated by researchers through varying approaches. Currently, most studies focusing on liver CSCs rely on cell surface markers, such as $\mathrm{CD}_{13}(1,2)$, $\mathrm{OV}_{6}(3), \mathrm{CD}_{133}$ (4), $\mathrm{CD}_{90}$ (5), $\mathrm{ABCG}_{2}$ (6) and EpCAM (7). This approach identified stem-like cancer cells with clonogenic and tumorigenic capacity, strongly supporting the existence of CSCs in HCC. However, different markers from different researchers make it confusing to understand liver CSC. Alternatively, SP approach, which is based on the functional property of CSCs to exclude Hoechst 33342 dye via ABCG2transporters, might have certain advantages for prospective isolation and characterization of CSCs (8-11). The SP assay requires an incubation step for appropriate equilibration of the dye Hoechst 33342 between the extracellular and intracellular compartments prior to dye efflux by cells expressing $\mathrm{ABC}$ transporters. ABC transporter-mediated dye efflux is an active and dynamic biological process and thus highly sensitive to even slight modifications, leading to discrepancies in the SP percentage (12). Therefore, it is critical to optimize Hoechst 33342 staining procedure to achieve a consistent and tumorigenic SP subtype.

Sphere-forming assays have been widely used to retrospectively identify stem cells based on their capacity to evaluate self-renewal and differentiation in vitro (13). The sphere-forming assay has also been applied to cultivate various CSCs. Uchida et al (14) demonstrated sphere-forming cells in a variety of live cancer cell lines and confirmed the stemness in these cells.

More and more studies have shown involvement of several miRNAs in the regulation of CSCs in the past a few years (15). In liver cancer, the miR-181 is important for the maintenance of hepatic CSC by posttranslational downregulation of two hepatic transcriptional regulators of differentiation and an inhibitor of Wnt/ $\beta$-catenin signaling (16). Li et al compared the miRNA profiles between rat liver CSC and normal liver stem cells, his study suggest that liver CSCs may have a distinct 
miRNA expression fingerprint (17). Moreover, a relationship between CSC and epithelial-mesenchymal transition (EMT) has recently emerged with evidence suggesting that EMT cells have cancer stem cell-like features and CSCs exhibit a mesenchymal-like phenotype (18). Many miRNAs are shown to be involved in both processes. For example, MiR-194 can target BMI-1 which is a self-renewal gene to inhibit EMT in endometrial cancer $(19,20)$. The miR-125b recently was found to be necessary for stem cell fission and make stem cells insensitive to chemotherapy (21). Also miR-125b can regulate EMT process by directly targeting oncogene LIN28B2 (22). Given the strong link between miRNAs and CSCs, this study focuses on an accurate identification of liver CSC and CSC- specific miRNAs.

\section{Materials and methods}

Cell lines and animals. HCC cell line PLC/PRF/5, Huh-7 and Hep-3B were purchased from Shanghai Cell Center. PLC/ PRF/5 cells were cultured in MEM (Hyclone, Logan, UT, USA) containing $10 \%$ fetal bovine serum (FBS, Hyclone), while Huh-7 and Hep-3B in PRMI-1640 (Hyclone) with $10 \%$ FBS. Cells were incubated at $37^{\circ} \mathrm{C}$ in a humidified atmosphere containing $5 \% \mathrm{CO}_{2}$. Male non-obese diabetic/severe combined immunodeficiency (NOD/SCID) mice (6-8 weeks) were purchased from Shanghai Institute of Material Medicine, Chinese Academy of Science and housed in lamina flow cabinets under specific pathogen-free conditions. The experiments were done in accordance with our institutional guidelines for the use of laboratory animals.

Hoechst 33342 staining optimization. The cultured cells with $75 \%$ confluence were detached with $0.25 \%$ trypsin-EDTA and suspended at $1 \times 10^{6} \mathrm{cells} / \mathrm{ml}$ in fresh medium. Cells were counted using trypan blue at least twice to ensure an identical staining density $\left(1 \times 10^{6}\right.$ cells $\left./ \mathrm{ml}\right)$. The cells collected at different culture times were then incubated with different concentrations of Hoechst 33342 (Invitrogen, Carlsbad, CA, USA) either alone or in the presence of $50 \mu \mathrm{g} / \mathrm{ml}$ verapamil (Sigma-Aldrich, St. Louis, MO, USA) at $37^{\circ} \mathrm{C}$ for 60 or 90 or 120 min with different shaking intervals. The 4 factors, incubation time, shaking interval, culture time and dye concentration, were all tested independently. Verapamil was traditionally used as a guiding parameter to determine the boundary between SP and NSP cells. Samples were then washed, centrifuged and resuspended in $1 \mathrm{ml}$ cold PBS supplemented with 3\% FBS. Propidium iodide (PI, Sigma-Aldrich) was added at $1 \mu \mathrm{g} / \mathrm{ml}$ to exclude the dead cells before FCM analysis. Each assay was done in triplicate.

MoFlo XDP analysis and sorting strategy. MoFlo XDP was used to analyze and sort SP and NSP cells. The Hoechst 33342 was excited with the UV laser at $350 \mathrm{~nm}$ and fluorescence emission was measured with 450 (Hoechst blue) and 675 (Hoechst red) optical filters. When sorting, MoFlo XDP checkup was done each time to ensure accuracy and SP gate was also refined. Sorted cells were placed on ice to increase viability and then stored in RNAprotect Cell Reagent (Qiagen, Hilden, Germany) for further isolation of high quality miRNAs. The purity of sorted cells was also analyzed.
Floating sphere cultivation. To culture floating spheres, SP and NSP cells sorted from the three cell lines were re-suspended respectively, in serum-free medium (SFM) containing DMEM/F12 (Hyclone) supplemented with $20 \mathrm{ng} / \mathrm{ml}$ EGF (Peprotech, Princeton, NJ, USA), $10 \mathrm{ng} / \mathrm{ml}$ bFGF (Peprotech), $5 \mu \mathrm{g} / \mathrm{ml}$ insulin (Upstate, NY, USA) and $10 \mu \mathrm{l} / \mathrm{ml} \mathrm{B27} \mathrm{(PAA,}$ Pasching, Austria), $1 \times 10^{4}$ cells/well in 6-well plates. Cells were then incubated at $37^{\circ} \mathrm{C}$ in a humidified atmosphere containing $5 \% \mathrm{CO}_{2}$.

Tumorigenicity assay in vivo. To explore the tumorigenic capacity, SP and NSP cells sorted from the three HCC cell lines, suspended in $200 \mu \mathrm{l}$ DMEM and Matrigel (1:1, BD Biosciences, San Jose, CA, USA), were injected into the right and left axila of NOD/SCID mice at a total number of $10,1 \times 10^{2}, 1 \times 10^{3}$ and $1 \times 10^{4}$ cells, respectively. Tumor formation was monitored weekly after implantation. Animals were sacrificed after 12 weeks. Each group contained 5 animals in tumor initiation experiment.

MiRNA profiling by $q P C R$ assay. Freshly isolated three batches of SP and NSP cells were kept in RNAprotect Cell Reagent and shipped to Beijing Genomics of Sciences for miRNA isolation and qPCR assay. Total RNAs were then obtained by miRNeasy Micro kit. The extracted RNAs were evaluated with Nanadrop 8000 and agarose gel electrophoresis to ensure its quality. Three steps-based qPCR method was then adopted. A-Plus bacterial polyA polymerase was used to add polyA tail to mature form of miRNAs and reverse transcription (RT) was done for first strand cDNA synthesis. Subsequently SYBR Green-based qPCR reaction was conducted on ABI 7500 (Life Technologies, Foster City, CA, USA) under optimized conditions. A total of 370 miRNAs were tested using primers provided by Beijing Genomics of Sciences. U6 was used as an internal control during the process of RNA isolation, poly(A) plus, cDNA synthesis and qPCR. Second qPCR quality control was used during the qPCR procedure. The $\mathrm{C}_{\mathrm{T}}$ values of 370 miRNAs were evaluated and the data were shown as fold changes $\left(2^{-\Delta \Delta \mathrm{C}_{\mathrm{T}}}\right)$ to analyze the miRNA expression levels. The results were normalized against U6 and t-test was used for comparison among groups. $\mathrm{P}<0.05$ was considered statistically significant.

MiRNA verification by $q P C R$ assay. To evaluate whether the profiling result is univerval across differential HCC cell lines, the differential miRNAs were tested again in SP and NSP cells from Huh-7 and Hep-3B cell lines. The qPCR procedures were the same as stated above.

\section{Results}

$S P$ staining optimization and MoFlo XDP sorting. The 4 factors, incubation time, shaking interval, culture time and dye concentration were tested one by one when the other three were fixed. The optimal incubation time and shaking interval were determined by staining PLC/PRF/5 cells cultured for 2 days with $9 \mu \mathrm{g} / \mathrm{ml}$ of Hoechst 33342 for 60,90 and $120 \mathrm{~min}$ with $30 \mathrm{~min}$ of shaking interval and then for $90 \mathrm{~min}$ with 15 , 30 and 90 min of shaking interval, respectively. We found that 

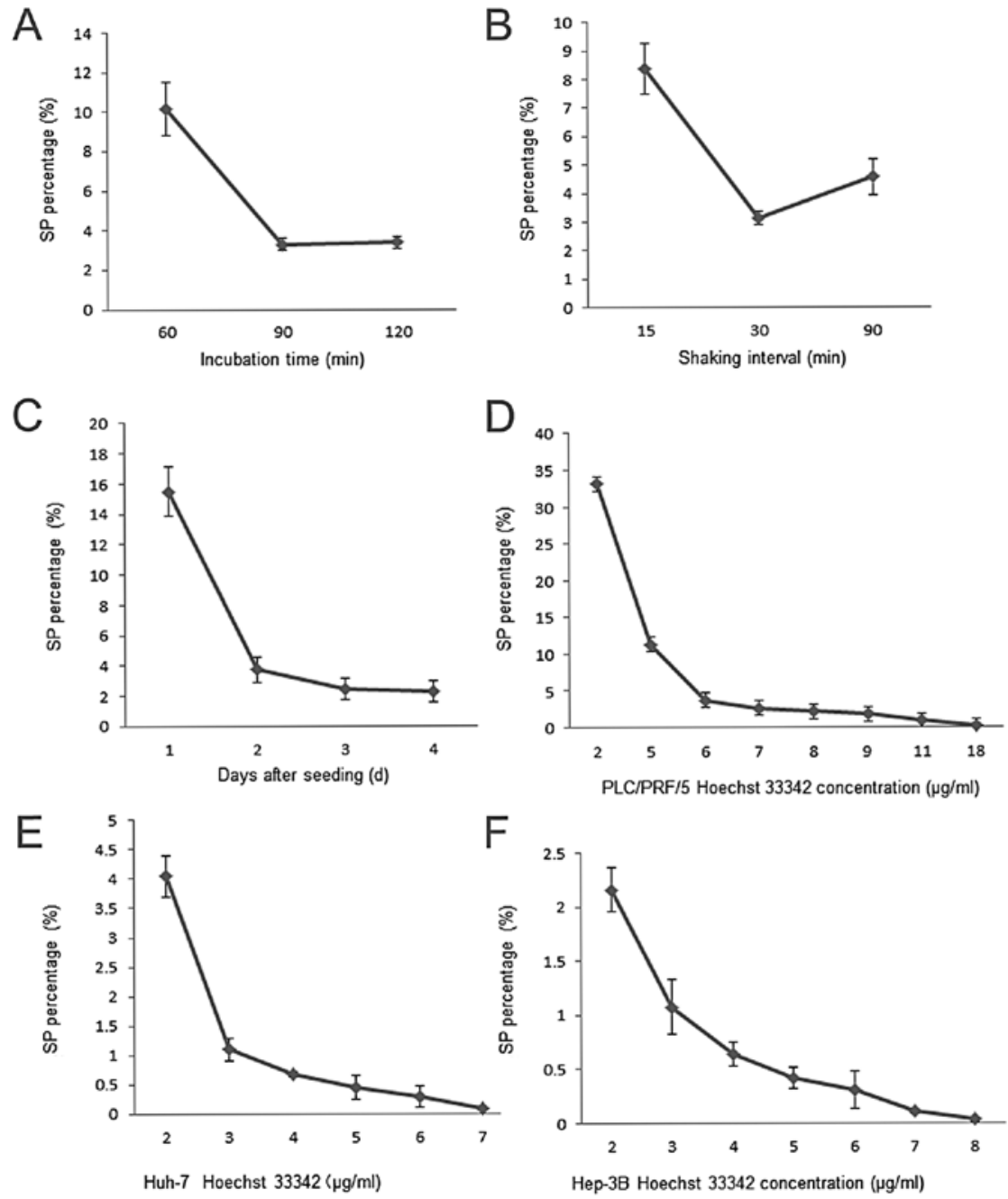

Figure 1. SP cell analysis: Hoechst 33342 staining condition optimization. (A) Incubation time: when (B-D) remain constant, an incubation time of 90-min produces the lowest SP percentage. (B) Shaking interval: when (A, C and D) remain constant, a shaking interval of 30 min produces the lowest SP percentage. (C) Culture time: when (A, B and D) remain constant, day 3 cells produce the lowest SP percentage. (D) Hoechst 33342 concentration, when (A-C) remain constant for PLC/PRF/5: a Hoechst 33342 concentration of $10 \mu \mathrm{g} / \mathrm{ml}$ produces an appropriate SP percentage. (E) Hoechst 33342 concentration when (A-C) remain constant for Huh-7: a Hoechst 33342 concentration of $4 \mu \mathrm{g} / \mathrm{ml}$ produces an appropriate SP percentage. (F) Hoechst 33342 concentration when (A-C) remain constant for Hep-3B: a Hoechst 33342 concentration of $5 \mu \mathrm{g} / \mathrm{ml}$ produces an appropriate SP percentage. N=3. Error bars represent standard deviation.

90-min incubation time produced a lower SP percentage, so did the 30-min shaking interval.

To determine the culture time, PLC/PRF/5 cells were seeded into $4 \mathrm{~T}-25$ flasks at $0.5 \times 10^{6}$ cells $/ \mathrm{ml}$ under the same conditions. The cells were cultured for up to 4 days and harvested at the end of day 1,2,3 and 4, respectively. They were then used to determine SP percentage under the standard staining protocol. The SP percentage was highest at day 1 and lowest at days 3 and 4 , indicating that the SP percentage reached plateau stage before day 3 (Fig. 1).

Different concentrations of Hoechst 33342 ranging from 2 to $18 \mu \mathrm{g} / \mathrm{ml}$ were used respectively, to stain day $3 \mathrm{PLC} / \mathrm{PRF} / 5$, Huh-7 as well as Hep-3B cells for 90-min incubation with 30 -min shaking interval. The results showed that dye concentration exerted a pronounced effect on SP\%. As Hoechst concentration decreased, SP percentages also decreased, first sharply then slightly, suggesting the optimal dye concentration for staining is $11 \mu \mathrm{g} / \mathrm{ml}$ for PLC/PRF $/ 5,4 \mu \mathrm{g} / \mathrm{ml}$ for Huh-7 and $5 \mu \mathrm{g} / \mathrm{ml}$ for Hep-3B cells (Fig. 1).
Under the optimized procedure, the resultant SP percentage was $0.73 \pm 0.12 \%$ in PLC/PRF/5 cells, $0.49 \pm 0.04 \%$ in Huh-7 and $0.63 \pm 0.08 \%$ in Hep-3B cells. The purity of sorted SP cells was $>85 \%$ (Fig. 2).

Floating sphere assay. To test CSC characteristics, SP and NSP cells were sorted out to cultivate in SFM. Many sorted SP cells were able to survive in SFM. On day 4, sorted SP cells were beginning to propagate into floating cells. NSP cells could not form a single floating sphere and eventually died. When the spheres were dispersed into single cells, secondary and tertiary spheres also formed. Of the three cell lines, Hep-3B SP cells formed the largest floating spheres, while Huh-7 the smallest (Fig. 3, SP x400).

Tumor formation assay in NOD/SCID mice. To determine whether the SP cells were more tumorigenic than NSP cells, the two types of cells from each of the three cell lines were inoculated s.c. into the axillae of NOD/SCID mice at different 

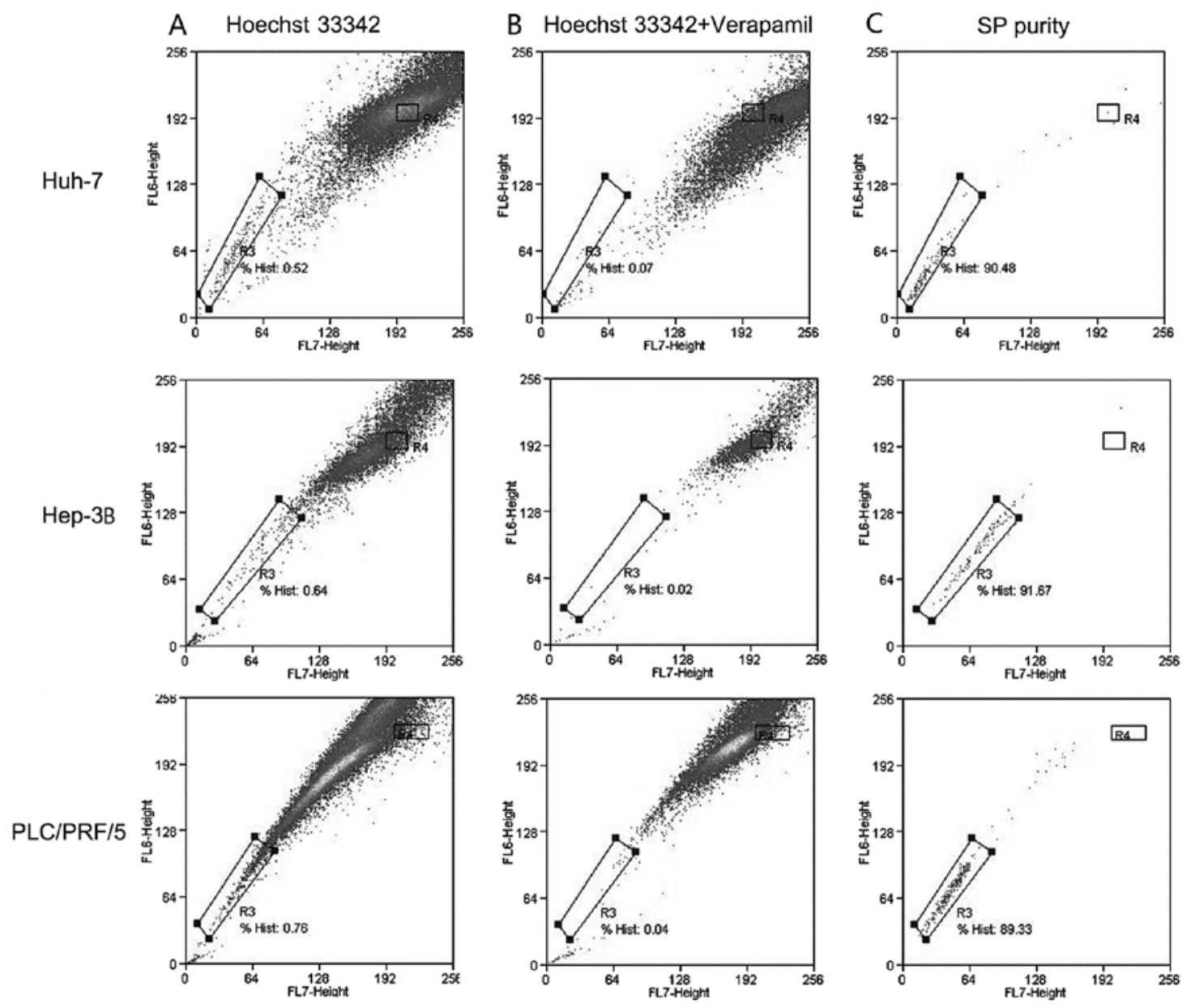

Figure 2. SP percentages in PLC/PRF/5 cell line after optimization of staining and sorting purity. (A) Staining by Hoechst 33342 alone, the resultant SP percentage after staining optimization for the three cell lines. (B) Staining by Hoechst $33342+$ verapamil, The SP cells disappeared with Hoechst 33342 and verapamil co-treatment. (C) SP purity was $>85 \%$.
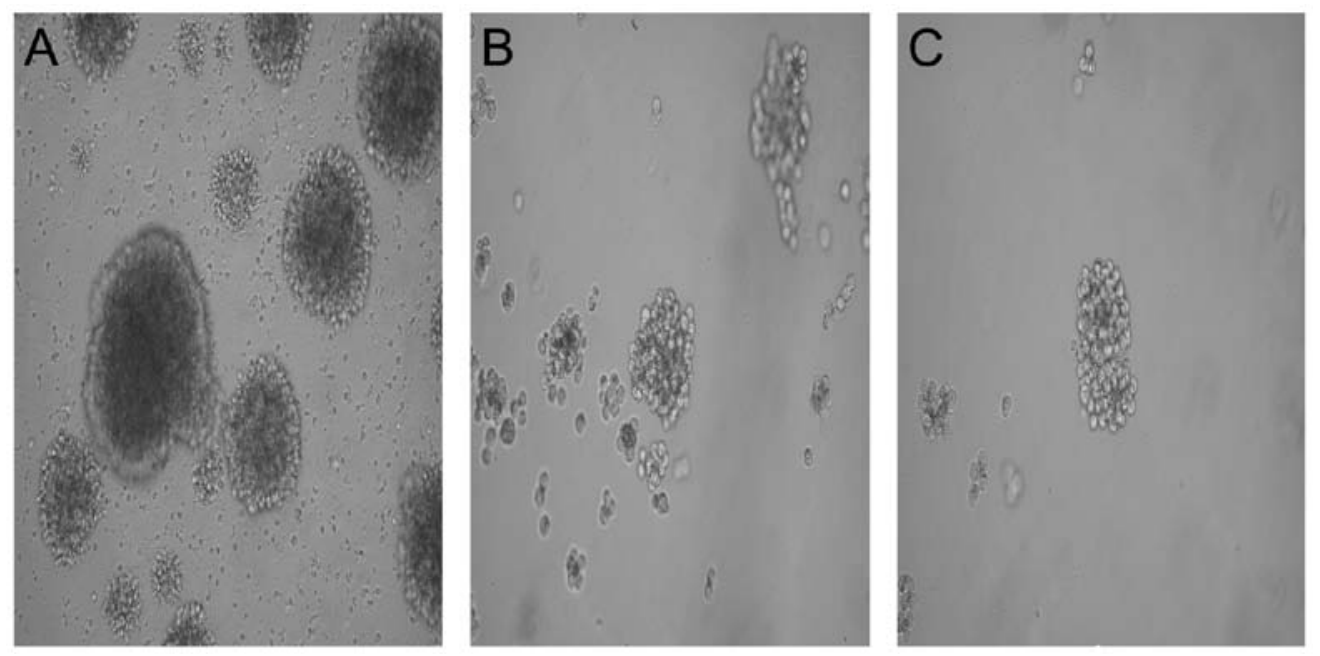

Figure 3. Floating spheres formed by three hepatocellular cell lines on day 6. (A) Floating spheres formed by SP cells from Hep-3B. (B) Floating spheres formed by SP cells from PLC/PRF/5. (C) Floating spheres formed by SP cells from Huh-7.

cell concentrations, respectively, with SP cells in the left and NSP the right side. The mice were sacrificed 12 weeks later. When 10 cells were injected, neither SP nor NSP cells could form tumors. At $1 \times 10^{4}$ cells, all SP cells could form tumors in the three cell lines, while NSP cells formed fewer tumors. The larger amount of injected cells was, the more tumor masses 


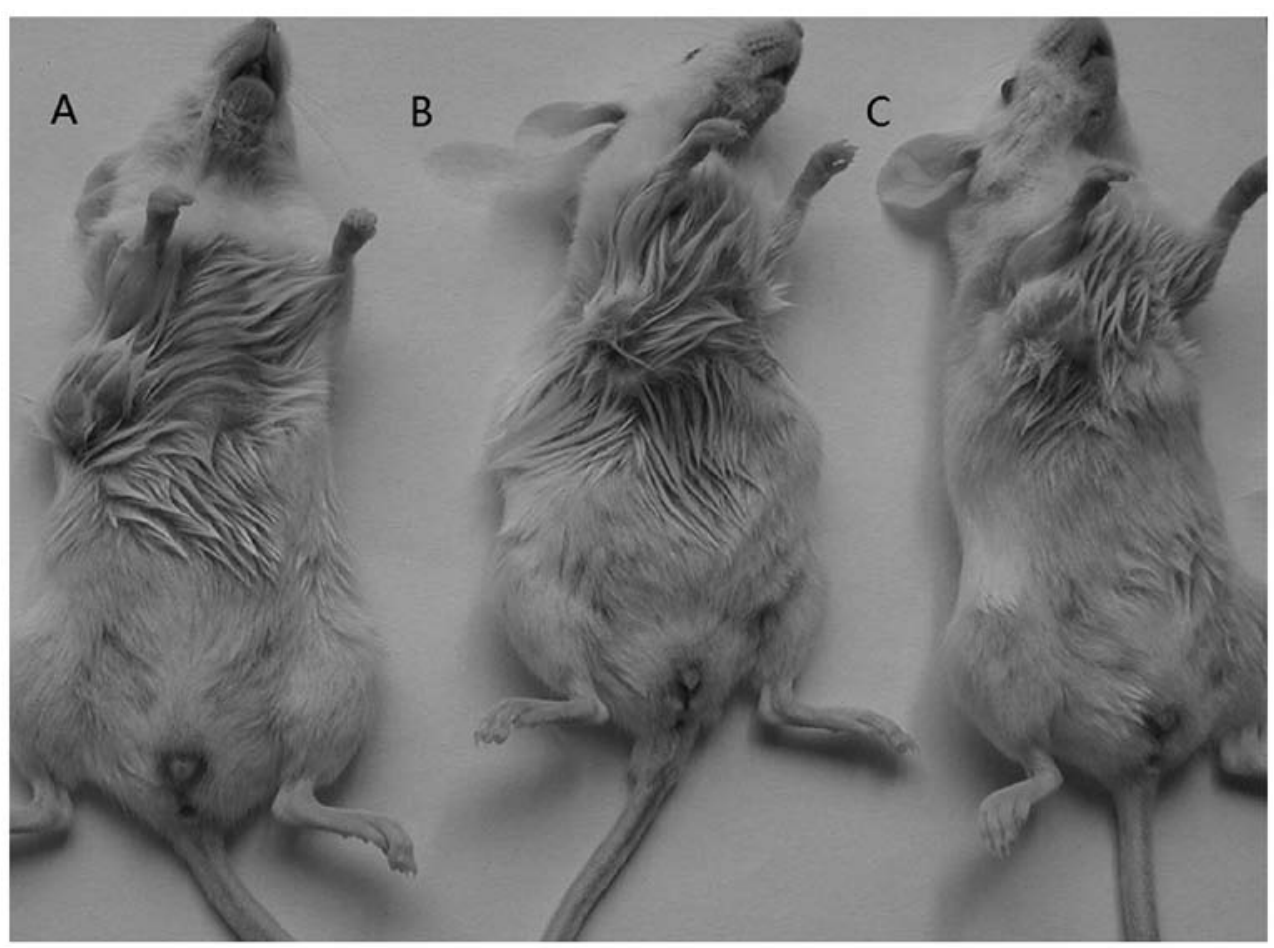

Figure 4. Tumors formed at week 12 by $1 \times 10^{3}$ cells in both sides. (A) Mouse injected with PLC/PRF/5 cells: 1 tumor was formed on the right side by SP cells while no tumor by NSP on the left. (B) Mouse injected with Huh-7 cells: 1 tumor was formed on the right side by SP cells while no tumor by NSP on the left. (C) Mouse injected with Hep-3B cells: 1 large tumor was formed on the right side by SP cells and 1 small tumor by NSP on the left.

Table I. Tumors formed in NOD/SCID mice $(n=5)$.

\begin{tabular}{llcccc}
\hline & & \multicolumn{4}{c}{ Tumor formed } \\
\cline { 3 - 6 } Cell line & Group & 10 & $1 \times 10^{2}$ & $1 \times 10^{3}$ & $1 \times 10^{4}$ \\
\hline PLC/PRF/5 & SP cells & $0 / 5$ & $1 / 5$ & $5 / 5$ & $5 / 5$ \\
& NSP cells & $0 / 5$ & $0 / 5$ & $0 / 5$ & $2 / 5$ \\
Huh-7 & SP cells & $0 / 5$ & $1 / 5$ & $4 / 5$ & $5 / 5$ \\
& NSP cells & $0 / 5$ & $0 / 5$ & $1 / 5$ & $1 / 5$ \\
Hep-3B & SP cells & $0 / 5$ & $2 / 5$ & $5 / 5$ & $5 / 5$ \\
& NSP cells & $0 / 5$ & $0 / 5$ & $1 / 5$ & $2 / 5$ \\
\hline
\end{tabular}

SP and NSP cells were isolated separately and injected into the axillae of NOD/SCID mice. Tumor formation was observed for 12 weeks after injection.

were produced. Mice injected with SP cells on the right side formed more tumor masses than their NSP counterparts did (Table I and Fig. 4).

MiRNA qPCR expression profiling in SP and NSP samples. MiRNA expression of both SP and NSP cells from PLC/PRF/5 cell line was tested by 3 step-based qPCR method. MiRNAs with fold changes $(\mathrm{SP} / \mathrm{NSP})>2$ or $<0.5(\mathrm{P}<0.05)$ were selected. A total of 370 miRNA species were analyzed in 3 batches of SP and NSP. By the criteria stated above, 27 miRNAs were identified as differentially expressed, all of which were downregulated in SP (Table II).

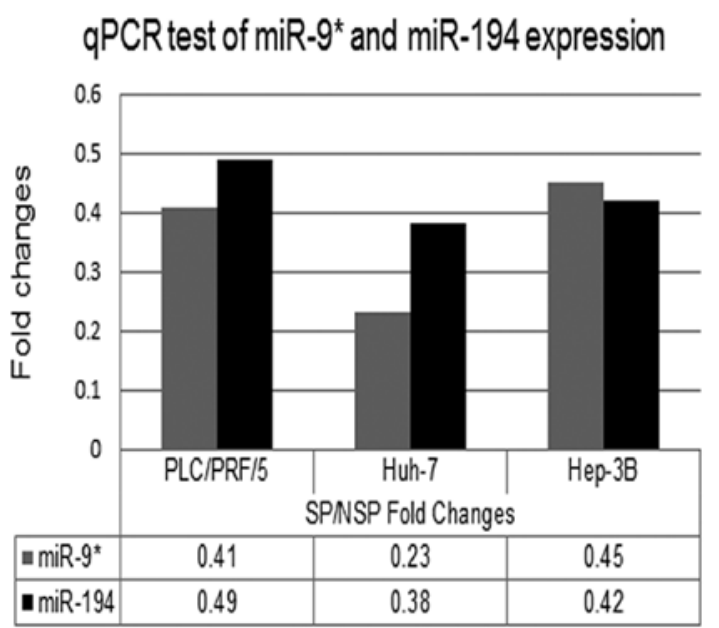

Figure 5. qPCR test of miR-9* and miR-194 expression in three cell lines.

MiRNA verification by $q P C R$ assay. MiRNA expressions of the 27 differential miRNAs were further tested in both the SP and NSP cells from Huh-7 and Hep-3B cell lines by 3 stepbased qPCR method. MiRNAs with fold changes (SP/NSP) $>2$ or $<0.5(\mathrm{P}<0.05)$ were selected. By the criteria stated above, miR-9* and miR-194 were identified (Fig. 5).

\section{Discussion}

The CSCs can be identified and isolated by different methodologies, including isolation by CSC-specific cell surface marker expression, detection of SP phenotype by Hoechst 33342 exclu- 
Table II. Fold changes of differentially expressed miRNAs in SP samples vs NSP samples $(n=3)$.

\begin{tabular}{lcc}
\hline miRNA ID & P-value $(<0.05)$ & Fold changes (SP/NSP) \\
\hline miR-9 & 0.0027 & -3.00 \\
miR-9* & 0.0044 & -2.41 \\
miR-23b & 0.0025 & -2.12 \\
miR-27b & 0.0024 & -2.35 \\
miR-9*6 & 0.0077 & -2.80 \\
miR-151-3p & 0.0044 & -2.65 \\
miR-151-5p & 0.0042 & -2.57 \\
miR-183* & 0.0088 & -2.23 \\
miR-183 & 0.0082 & -3.16 \\
miR-194 & 0.0032 & -2.03 \\
miR-146a & 0.0073 & -3.44 \\
miR-148b & 0.0001 & -3.14 \\
miR-340-3p & 0.0001 & -2.85 \\
miR-421 & 0.0042 & -2.32 \\
miR-135b* & 0.0158 & -3.55 \\
miR-489 & 0.0380 & -3.28 \\
miR-15a & 0.028 & -2.79 \\
miR-21 & 0.0106 & -2.67 \\
miR-22 & 0.0331 & -2.50 \\
miR-365 & 0.0388 & -2.47 \\
miR-450a-5p & 0.0215 & -2.46 \\
miR-7a & 0.0158 & -2.44 \\
miR-26b & 0.0264 & -2.28 \\
miR-125a-5p & 0.0231 & -2.27 \\
miR-25 & 0.0118 & -2.22 \\
miR- 126-3p & 0.0329 & -2.15 \\
miR-182 & 0.0119 & -14 \\
\hline
\end{tabular}

Three SP and NSP samples were tested by 3 step-based qPCR method for miRNA expression. MiRNAs with fold changes (SP/NSP) $>2$ or $<0.5(\mathrm{P}<0.05)$ were selected. Twenty-seven miRNAs were identified as differentially expressed, all of which were downregulated in SP.

sion, assessment of their ability to grow as floating spheres and aldehyde dehydrogenase (ALDH) activity assay. None of the methods mentioned are exclusively used to isolate the solid tumor CSCs, highlighting the need to delineate more specific markers or to use combinatorial markers and methodologies (23). Therefore, we combined SP phenotype and floating sphere formation methods to identify liver CSC.

In the SP phenotype assay, we firstly examined the 4 factors involved in the actual staining procedure and the results showed that all 4 factors contributed significantly to changes in SP percentage. The optimal Hoechst 33342 concentrations were determined when the other three conditions were fixed. As Hoechst- 33342 concentration decreased, cells were shifted from the NSP to SP region (24). Therefore the optimal Hoechst 33342 concentration should be chosen within a plateau region of the curve with complete verapamil inhibition. Apart from the 4 factors mentioned above, cell viability, storage time of Hoechst 33342 solution and even water bath temperature may result in variations in SP percentage. As long as the SP cells are limited to only verapamil-sensitive cells with the lowest Hoechst 33342 incorporation, the obtained SP is more homogeneous.

Many researchers have also proved the existence of SP in these cell lines with slightly different SP percentages from ours (8-10). However, they adopted only the SP method to define CSC. CSC can grow as non-adherent spheres. Uchida et al demonstrated sphere formation in HepG2, Hep3B and PLC/PRF/5 cells, but not in SK-Hep1 and Huh-7 cells (14). In our study, Huh-7, Hep3B and PLC/PRF/5 all formed floating spheres. The difference might be in the starting cells. We used SP cells to cultivate floating spheres, while Uchida et al used the whole population. Our assay proves that SP cells are the only cells with spheres-forming ability.

We then compared the tumorigenic ability of SP and NSP cells by assessing tumor formation in NOD/SCID mice, the gold standard for proving the existence of cancer stem cells despite its limitations. The assay confirmed a stronger proliferative and tumorigenic ability with SP cells, suggesting that our SP staining and isolation methods are eligible for isolating liver CSC.

MiRNAs regulate self-renewal, differentiation and division of cells via post-transcriptional gene silencing. Aberrant miRNA levels, specifically an overall downregulation, are present in many cancers and CSCs (25). Our study identified 27 downregulated miRNAs in SP cells from PLC/PRF/5 cell line. Among them, many are involved in the process of selfrenewal and metastasis in various cancers. miR-9* (26) and Let-7 family (27) are reported to impart stemness potential via their downregulation in breast cancer and glioma cells, respectively. MiR-194 (19,20) and miR-125b (21,22) can regulate the EMT and CSC processes in hepatocellular carcinoma, endometrial and breast cancer. MiR-146 can suppress breast cancer metastasis (28). All these data to some extent suggest that $\mathrm{SP}$ cells from $\mathrm{PLC} / \mathrm{PRF} / 5$ cell line possess CSC characteristics.

To evaluate whether the 27 miRNAs are universally downregulated across different $\mathrm{HCC}$ cell lines, their expression were further tested in both SP and NSP cells from Huh-7 and Hep-3B cell lines. Of all the 27 miRNAs, miR-9* and miR-194 were also shown to be downregulated, suggesting the miR-9* and miR-194 might be critical in maintaining the liver CSC ability. MiR-9* and miR-194 are both reported to regulate EMT and CSC via different mechanism in different cancers, but their functions in liver cancer are not fully explored. Our future studies will focus on the function of $\operatorname{miR}-9^{*}$ and miR-194 in hepatic carcinogenesis as well as their expression in tumor specimens, in the hope of finding a new cure for HCC patients.

\section{Acknowledgements}

This study was supported by Natural Science Foundation of Fujian Province (no. 2008J0077) and (no. 2012J05138). We thank Professor Shiyou Li from the Beijing Institute of Genomics for support of miRNA qPCR profiling. 


\section{References}

1. Haraguchi N, Ishii $\mathrm{H}$, Mimori $\mathrm{K}$, et al: $\mathrm{CD} 13$ is a therapeutic target in human liver cancer stem cells. J Clin Invest 120 $3326-3339,2010$

2. Kim HM, Haraguchi N, Ishii H, et al: Increased CD13 expression reduces reactive oxygen species, promoting survival of liver cancer stem cells via an epithelial-mesenchymal transition-like phenomenon. Ann Surg Oncol 19: 539-548, 2011.

3. Yang W, Wang C, Lin Y, et al: OV6(+) tumor-initiating cells contribute to tumor progression and invasion in human hepatocellular carcinoma. J Hepatol 57: 613-620, 2012.

4. Piao LS, Hur W, Kim TK, et al: CD133+ liver cancer stem cells modulate radioresistance in human hepatocellular carcinoma. Cancer Lett 315: 129-137, 2011.

5. Lingala S, Cui YY, Chen X, et al: Immunohistochemical staining of cancer stem cell markers in hepatocellular carcinoma. Exp Mol Pathol 89: 27-35, 2010.

6. Sukowati CH, Rosso N, Pascut D, et al: Gene and functional up-regulation of the BCRP/ABCG2 transporter in hepatocellular carcinoma. BMC Gastroenterol 12: 160, 2012.

7. Yamashita T, Ji J, Budhu A, et al: EpCAM-positive hepatocellular carcinoma cells are tumor-initiating cells with stem/progenitor cell features. Gastroenterology 136: 1012-1024, 2009.

8. Shi GM, Xu Y, Fan J, et al: Identification of side population cells in human hepatocellular carcinoma cell lines with stepwise metastatic potentials. J Cancer Res Clin Oncol 134: 1155-1163, 2008.

9. Ma S, Chan KW, Hu L, et al: Identification and characterization of tumorigenic liver cancer stem/progenitor cells. Gastroenterology 132: 2542-2556, 2007.

10. Chiba T, Miyagi S, Saraya A, et al: The polycomb gene product BMI1 contributes to the maintenance of tumor-initiating side population cells in hepatocellular carcinoma. Cancer Res 68 : 7742-7749, 2008

11. Chiba T, Kita K, Zheng YW, et al: Side population purified from hepatocellular carcinoma cells harbors cancer stem cell-like properties. Hepatology 44: 240-251, 2006.

12. Golebiewska A, Brons NH, Bjerkvig R and Niclou SP: Critical appraisal of the side population assay in stem cell and cancer stem cell research. Cell Stem Cell 8: 136-147, 2011.

13. Pastrana E, Silva-Vargas V and Doetsch F: Eyes wide open: a critical review of sphere-formation as an assay for stem cells. Cell Stem Cell 8: 486-498, 2011.
14. Uchida Y, Tanaka S, Aihara A, et al: Analogy between sphere forming ability and stemness of human hepatoma cells. Oncol Rep 24: 1147-1151, 2010.

15. Zimmerman AL and Wu S: MicroRNAs, cancer and cancer stem cells. Cancer Lett 300: 10-19, 2006.

16. Ji J, Yamashita T, Budhu A, et al: Identification of microRNA-181 by genome-wide screening as a critical player in EpCAM-positive hepatic cancer stem cells. Hepatology 50: 472-480, 2009.

17. Li R, Qian N, Tao K, et al: MicroRNAs involved in neoplastic transformation of liver cancer stem cells. J Exp Clin Cancer Res 29: $169,2012$.

18. DiMeo TA, Anderson K, Phadke P, et al: A novel lung metastasis signature links Wnt signaling with cancer cell self-renewal and epithelial-mesenchymal-transition in basal-like breast cancer Cancer Res 69: 5364-5373. 2009.

19. Meng Z, Fu X, Chen X, et al: miR-194 is a marker of hepatic epithelial cells and suppresses metastasis of liver cancer cells in mice. Hepatology 52: 2148-2157, 2010.

20. Dong P, Kaneuchi M, Watari H, et al: MicroRNA-194 inhibits epithelial to mesenchymal transition of endometrial cancer cells by targeting oncogene BMI-1. Mol Cancer 10: 99, 2011.

21. Shi L, Zhang J, Pan T, et al: MiR-125b is critical for the suppression of human U251 glioma stem cell proliferation. Brain Res 1312: $120-126,2010$

22. Liang L, Wong CM, Ying Q, et al: MicroRNA-125b suppressesed human liver cancer cell proliferation and metastasis by directly targeting oncogene LIN28B2. Hepatology 52: 1731-1740, 2012.

23. Tirino V, Desiderio V, Paino F, et al: Cancer stem cells in solid tumors: an overview and new approaches for their isolation and characterization. FASEB J 27: 13-24, 2013.

24. Mo SL, Li J, Loh YS, et al: Factors influencing the abundance of the side population in a human myeloma cell line. Bone Marrow Res 52: 2148-2157, 2011.

25. Mott JL: MicroRNAs involved in tumor suppressor and oncogene pathways: implications for hepatobiliary neoplasia. Hepatology 50: 630-637, 2009

26. Jeon HM, Sohn YW, Oh SY, et al: ID4 imparts chemoresistance and cancer stemness to glioma cells by derepressing miR-9*-mediated suppression of SOX2. Cancer Res 71: 3410-3421, 2011.

27. Yu F, Yao H, Zhu P, et al: let-7 regulates self- renewal and tumorigenicity of breast cancer cells. Cell 131: 1109-1123, 2007.

28. Hurst DR, Edmonds MD, Scott GK, et al: Breast cancer metastasis suppressor 1 up-regulates miR-146, which suppresses breast cancer metastasis. Cancer Res 69: 1279-1283. 2009. 